\title{
Fingerprint and On-Line Signature Verification Competitions at ICB 2009
}

\author{
Bernadette Dorizzi $^{1}$, Raffaele Cappelli ${ }^{2}$, Matteo Ferrara ${ }^{2}$, Dario Maio ${ }^{2}$, \\ Davide Maltoni ${ }^{2}$, Nesma Houmani ${ }^{1}$, Sonia Garcia-Salicetti ${ }^{1}$, and Aurélien Mayoue ${ }^{1}$ \\ ${ }^{1}$ Institut TELECOM; TELECOM \& Management SudParis, Evry, France \\ ${ }^{2}$ Biometric System Laboratory, DEIS, University of Bologna, Italy \\ \{cappelli, ferrara, maio, maltoni\} acsr.unibo.it, \\ \{Bernadette.Dorizzi, Nesma.Houmani, \\ Sonia.Salicetti, Aurelien.Mayoue\}@it-sudparis.eu
}

\begin{abstract}
This paper describes the objectives, the tasks proposed to the participants and the associated protocols in terms of database and assessment tools of two competitions on fingerprints and on-line signatures. The particularity of the fingerprint competition is to be an on-line competition, for evaluation of fingerprint verification tools such as minutiae extractors and matchers as well as complete systems. This competition will be officialy launched during the ICB conference. The on-line signature competition will test the influence of multisessions, environmental conditions (still and mobility) and signature complexity on the performance of complete systems using two datasets extracted from the BioSecure database. Its result will be presented during the ICB conference.
\end{abstract}

Keywords: Fingerprint Verification, On-line signature Verification, Technical Evaluations, Signature Categorization, Entropy.

\section{Introduction}

Conducting biometric technical evaluations is important in order to stimulate research in the challenging and open issues left unsolved in the biometrics at hand. Comparative evaluations also provide to the stakeholders, information about the state of the art of the algorithms and the progress of the technology as well as tools such as databases and protocols for future benchmarking.

In this paper, we concentrate on two evaluations, which are now open in fingerprint and on-line signature. Their results will be ready for a presentation at the ICB 2009 conference. The first competition, FVC-onGoing is in the continuity of the FVCs competitions (Fingerprint Verification Competition) organized by University of Bologna in the past years. The second one, BSEC 2009 (BioSecure Signature Evaluation Campaign) follows the road opened last year by BMEC 2009 (BioSecure Multimodal Evaluation Campaign) except that this year, it is focused on one modality only, namely on-line signature and it will use the newly available BioSecure Database.

The rest of the paper describes in two separate sections (Section 2 and 3 ) the two competitions, their objectives, the tasks proposed to the participants and the associated protocols in terms of database and assessment tools. 


\section{FVC-onGoing}

Performance evaluation is important for all biometric modalities and particularly so for fingerprint recognition, which is receiving widespread attention for citizen identity verification and identification in large-scale applications. Unambiguously and reliably assessing the current state of the fingerprint recognition technology is mandatory for understanding its limitations and addressing future research.

After the success of the first four fingerprint verification competitions (FVC2000 [1], FVC2002 [2], FVC2004 [3] and FVC2006 [4]), the Biometric System Laboratory (University of Bologna) has decided to organize a new online evaluation campaign for fingerprint recognition technologies: FVC-onGoing.

FVC-onGoing will offer web-based automatic evaluation of fingerprint recognition algorithms on a set of sequestered datasets, reporting results using well known performance indicators and metrics.

The aim is to track the advances in fingerprint recognition technologies, through continuously updated independent testing and reporting of performances on given benchmarks. The benchmark datasets will not evolve over time; in case new datasets will be added in the future, they will form a different benchmark or a new version of an existing one: in this way, only results obtained on the same data will be compared.

The algorithms will be evaluated using strongly supervised approaches (see [3]), to maximize trustworthiness of the results.

While previous FVC initiatives were organized as "competitions", with specific calls and fixed time frames, FVC-onGoing will be:

- an "on going competition" always open to new participants;

- $\quad$ an evolving online repository of evaluation metrics and results.

Furthermore, the evaluation will be not only limited to fingerprint verification algorithms: ad hoc metrics and datasets for testing specific modules of fingerprint verification systems will be available. In fact, since results are always reported as FMR/FNMR values of the entire fingerprint recognition system developed, it is practically impossible to understand if an advancement in performance is due to a specific matching technique or is in large part due to a minor change in an existing feature extraction method. For example, the only way to objectively compare fingerprint matchers is to start from the same set of features (i.e., the set of minutiae for minutiae based matchers). This will allow to better understand the limits and the challenges not only of the whole recognition problem, but also of its building blocks, with clear benefits for researchers and algorithms' developers.

Benchmarks (with specific datasets and testing protocols) for the following (sub)problems are currently being developed, and others may be added in the future:

- Fingerprint Verification (assessment of the accuracy of one-to-one fingerprint matching algorithms);

- Orientation Image Extraction (assessment of the accuracy of orientation image extraction algorithms);

- Minutiae Extraction (assessment of the accuracy of minutiae extraction algorithms);

- Minutiae Matching (assessment of the accuracy of minutiae matching algorithms on datasets of minutiae templates). 
One of the main goals of FVC-onGoing is to fully automate the main steps of the evaluation: participant registration, algorithm submission, performance evaluation, and reporting of the results. To this purpose, the Biometric System Laboratory is currently developing a web-based evaluation framework, whose architecture and typical workflow are shown in Fig. 1. The development of this software system is expected to be completed by January 2009; during the first months of 2009, a beta testing phase will be carried out with some invited participants; the official start of FVC-onGoing is planned to be held in conjunction with ICB2009 (the reader may refer to [5] for the most updated information).

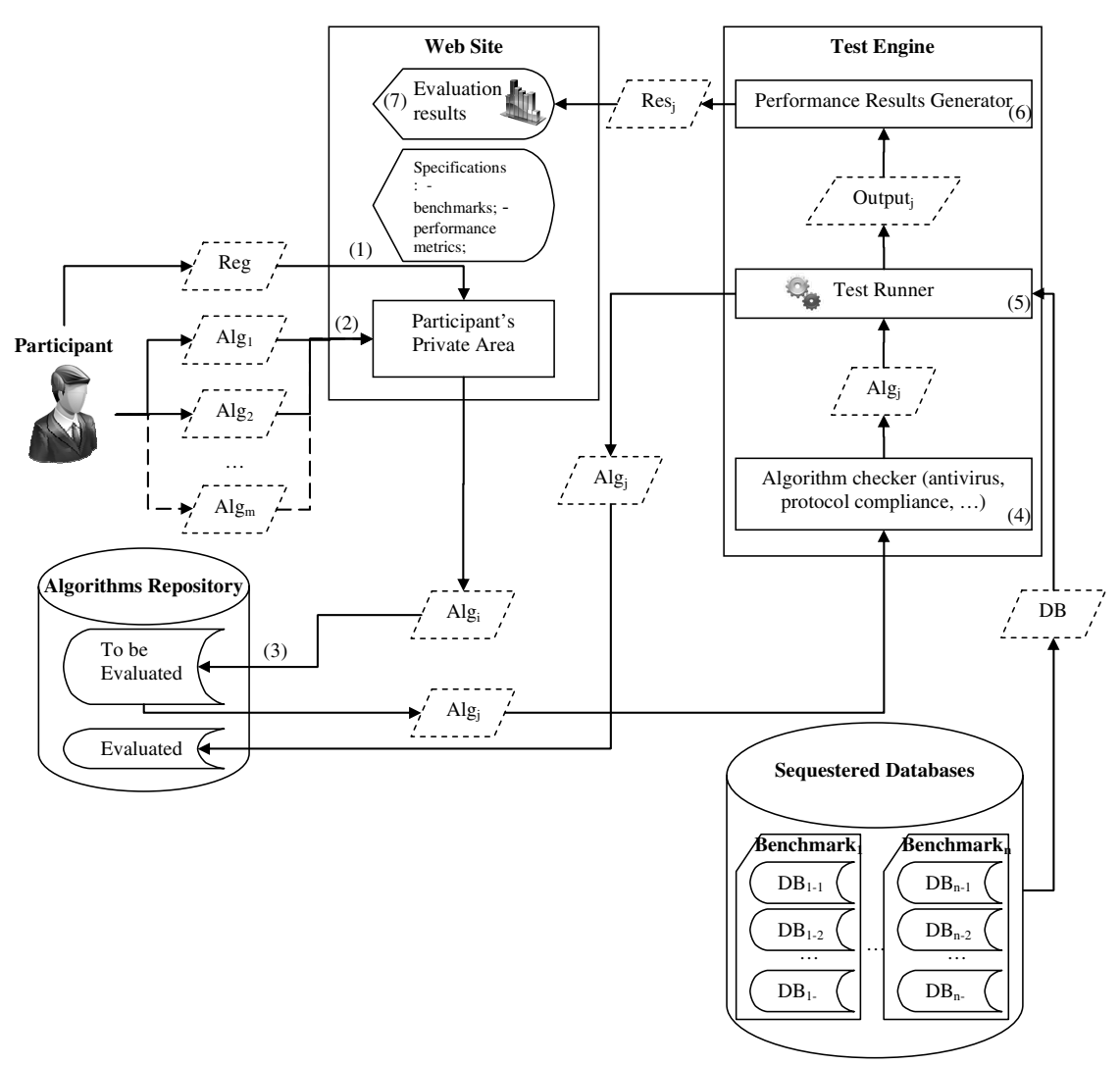

Fig. 1. The diagram shows the architecture of the FVC-onGoing evaluation framework and an example of a typical workflow: a given participant, after registering to the Web Site (1), submits some algorithms (2) to one or more of the available benchmarks; the algorithms (binary executable programs compliant to a given protocol) are stored in a specific repository (3). Each algorithm is evaluated by the Test Engine that, after some preliminary checks (4), executes it on the dataset of the corresponding benchmark (5) and processes its outputs (e.g. matching scores) to generate (6) all the results (e.g. EER, score graphs, ...), which are finally published (7) on the Web Site. 


\section{BSEC2009: The BioSecure Signature Evaluation Campaign}

\subsection{Motivation}

Pen-based interfaces on mobile platforms give nowadays another possible applicative context for signature verification algorithms. In the previous competitions, the First International Signature Verification Competition (SVC'2004) [6] and the BioSecure Multimodal Evaluation Campaign (BMEC'2007) [7,8], data came from a single sensor in each case: a digitizing tablet at SVC'2004 [6] (although 2 tasks with different parameters were tested) and a Personal Digital Assistant at BMEC'2007 [7, 8]. Also, in both cases performance was evaluated globally on all the persons of the database independently of any quality measured on the data. Finally, the impact of temporal variability was not studied at all in SVC'2004 and BMEC'2007; we thus aim in BSEC'2009 at doing a first step in this direction, by evaluating algorithms performance on two sessions spaced of 5 weeks.

BSEC2009 (BioSecure Signature Evaluation Campaign) [9] will be performed on the two existing largest databases containing the same persons, acquired on two different devices : indeed, BioSecure Signature Corpus DS3 and DS2 are the first on-line signature multisession databases acquired in a mobile scenario (on a PDA) for DS3, and on a digitizing tablet for DS2 [7,8]. BSEC2009 aims at measuring the real impact of a mobile platform on algorithms' performance using databases of large size. Another main goal of BSEC2009 is to evaluate algorithms' performance according to information content in signatures [9].

\subsection{Objectives}

The BioSecure Signature Evaluation Campaign has several objectives:

- To measure the real impact of mobility acquisition conditions on algorithms performance, by using the two largest existing online signature databases containing the same persons, BioSecure Signature Databases DS2 and DS3.

- To measure the impact of the information content of signatures on algorithms' performance, thanks to a protocol categorizing the data of both DS2 and DS3 in subsets. Indeed, performance will be measured by the evaluator not only globally (on the whole test database), but also on categories of signatures in terms of information content. To this end, we will exploit the notion of Client-Entropy, introduced in $[10,11]$ to categorize users depending on the quality of the signature (Complexity, Variability). This quality measure will not be available to participants; it will be only used by the evaluator in order to carry out performance assessment. In Section 3.3, we present in details the method provided by the organizer for categorizing the signatures.

- To do a first evaluation of the impact of time variability in the field.

\subsection{Entropy Measure and Client Categories}

\subsubsection{Measuring Client-Entropy with a Hidden Markov Model}

The entropy of a random variable only depends on its probability density values; thus, a good estimation of this probability density is important. As there are local dependencies in the dynamics of the hand-draw, a local paradigm for density estimation is natural. 
We consider each signature as a succession of portions, generated by its segmentation via the client-Hidden Markov Model (HMM) [12]. Therefore, we obtain as many portions in each signature as there are states in the client-HMM. Then we consider each point $(x, y)$ in a given portion as the outcome of one random variable $Z$ that follows a given probability mass function $p(z)=\operatorname{Pr}(Z=z)$ where $\mathrm{z}$ belongs to the Alphabet A of ordered pairs $(x, y)$.

Indeed, such random variable is discrete since its alphabet A has a finite number of values, nevertheless, the hand-draw as a time function is naturally a continuous process from which we retrieve a sequence of discrete values by the digitizer. For this reason, although $Z=(x, y)$ is discrete, its entropy in bits is defined as

$$
P(Z)=-\sum_{z \in A} p(z) \cdot \log _{2}(p(z))
$$

To compute the Client-Entropy measure, we first train the Client-HMM on 10 genuine signatures of such writer, after computing a personalized number of states, as follows:

$$
N=\frac{T_{\text {Total }}}{M * 30}
$$

where $T_{\text {Total }}$ is the total number of sampled points available in the genuine signatures, and $M=4$ is the number of Gaussian components per state. We consider that on average 30 sampled points are enough to estimate the mean vector and diagonal covariance matrix of each Gaussian component.

Then we exploit the Client-HMM to generate by the Viterbi algorithm the portions on which entropy is computed for each genuine signature. On each portion, we consider the probability density estimated by the Client-HMM to compute locally the entropy. We then average the entropy over all the portions of a signature and normalize the result by the signing time of the signature. Averaging this measure across the 10 genuine signatures on which the probability density was estimated by the HMM, allows generating a measure of the Client- Entropy. This measure is expressed in bits per second. Time-normalization allows comparing users between them in terms of entropy.

\subsubsection{Client Categories with Entropy Measure}

We performed on the two BioSecure Subsets DS2 and DS3 containing the same 382 persons, a $K$-Means on the Client- Entropy measure values for different values of $K$ and reached a good separation of signatures with $K=3$ as shown in Fig 1 and Fig 2, respectively on DS2 and DS3.

We notice visually that on the two Subsets of DS2 and DS3, the first category of signatures, those having the highest Client-Entropy measure, contains short, simply drawn and not legible signatures, often with the shape of a simple flourish. At the opposite, signatures in the third category, those of lowest Client-Entropy measure, are the longest and their appearance is rather that of handwriting, some are even legible. In between, we notice that signatures with medium Client-Entropy measure (second category) are longer and sometimes become legible, often showing the aspect of a complex flourish. 
(a)

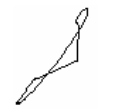<smiles></smiles><smiles>C1CCCC1</smiles><smiles>C1=CC1C1CC1</smiles><smiles>CCC(C)(C)C</smiles><smiles>C1CCCCC1</smiles>

(b)<smiles>CC#CCC</smiles><smiles>C1=CC2CCCC(C1)C2</smiles><smiles>[C]#[Co]</smiles><smiles>C#CC#CC(C)(C)C</smiles><smiles>C1C2C3CC2C13</smiles>

(c)
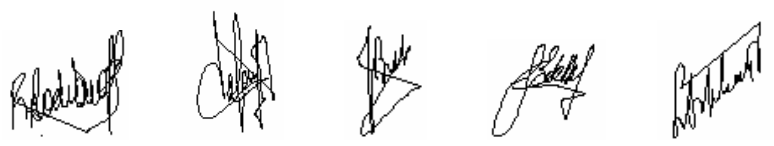

Fig. 1. Examples of signatures from DS2 of (a) highest, (b) medium and (c) lowest ClientEntropy measure

(a)

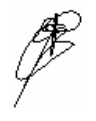

(b)

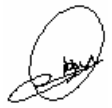

(c)

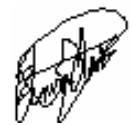

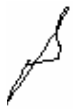
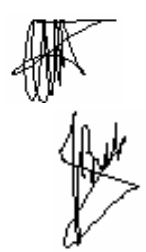
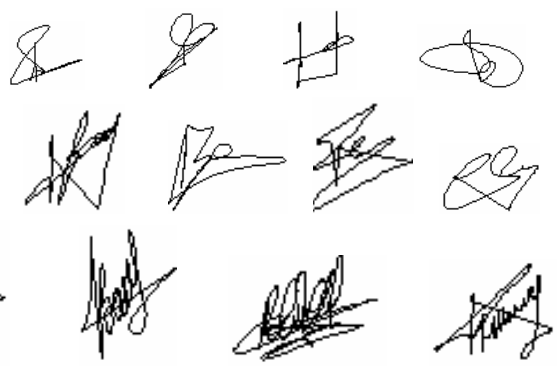

Fig. 2. Examples of signatures from DS3 of (a) highest, (b) medium and (c) lowest ClientEntropy measure

\subsection{Protocol and Databases}

\subsubsection{Description of Databases}

We use two databases in this evaluation: the two subsets of the Online Signature Databases acquired in the framework of the BioSecure Network of Excellence [7], DS3, acquired on a mobile platform (Personal Digital Assistant (PDA)) and DS2, acquired on a digitizer [8].

The whole BioSecure Signature Subcorpus DS3 and DS2 are not yet publicly available but, acquired on several sites in Europe, they are the first on-line signature multisession databases acquired in a mobile scenario (on a PDA) for DS3 and on a digitizer for DS2.

DS3 contains the signatures of 713 persons, acquired on the PDA HP iPAQ $\mathrm{hx} 2790$, at the frequency of $100 \mathrm{~Hz}$ and a touch screen resolution of $1280 * 960$ pixels. Three time functions are captured from the PDA: $x$ and y coordinates and the time elapsed between the acquisition of two successive points. The user signs while standing and has to keep the PDA in his or her hand. Two sessions were acquired spaced of around 5 weeks, each containing 15 genuine signatures. The donor was asked to perform, alternatively, three times five genuine signatures and twice five forgeries. For skilled forgeries, at each session, a donor is asked to imitate five times the signature of two other persons. In order to imitate the dynamics of the signature, the forger 
visualized on the PDA screen the writing sequence of the signature he/she had to forge and could sign on the image of such signature in order to obtain a better quality forgery both from the point of view of the dynamics and of the shape of the signature.

As original data acquired on the PDA have the property of variable time elapsed between two consecutive points captured, the organizer will perform time interpolation.

On the other hand, DS2 contains data from 667 persons acquired in a PC-based offline supervised scenario and the digitizing tablet WACOM INTUOS 3 A6. The pen tablet resolution is 5080 lines per inch and the precision is $0.25 \mathrm{~mm}$. The maximum detection height is $13 \mathrm{~mm}$ and the capture area is $270 \mathrm{~mm}$ (width) $\mathrm{x} 216 \mathrm{~mm}$ (height). Signatures are captured on paper using an inking pen. At each sampled point of the signature, the digitizer captures at $100 \mathrm{~Hz}$ sampling rate the pen coordinates, pen pressure (1024 pressure levels) and pen inclination angles (azimuth and altitude angles of the pen with respect to the tablet). This database contains two sessions, acquired two weeks apart. Fifteen (15) genuine signatures were acquired at each session as follows: the donor was asked to perform, alternatively, three times five genuine signatures and two times five skilled forgeries. For skilled forgeries, at each session, a donor is asked to imitate five times the signature of two other persons.

Two development sets of 50 users from respectively BioSecure Subsets DS2 and DS3 have been distributed to participants. Note that the 50 users are not the same in the two development sets.

Two test sets of 382 users from respectively BioSecure Subsets DS2 and DS3 are used and they will be kept sequestered. Note that the two test sets contain the same 382 users, in order to measure the real impact of mobility acquisition conditions on algorithms performance.

\subsubsection{Protocol}

Two tasks will be defined on DS2: Task1 considering the five available time functions captured by the digitizer (pen coordinates, pen pressure and pen inclination) and Task 2 with only pen coordinates.

For each individual enrolled in the signature database, we describe the enrolment and test sets valid for development sets as well as tests on sequestered data: five genuine signatures, acquired during the first session, are used as reference signatures for distance-based approaches and as training set for statistical approaches. The tests are done on the remaining 10 genuine signatures acquired during the first session, 15 genuine signatures from the second session, 20 skilled forgeries acquired during both sessions, and 30 random forgeries.

Submitted systems should compare each signature of the test set to the five signatures of the training set or to a model constructed from these five signatures. For each test, only one score should be provided by the system. This score must be normalized between 0 and 1 .

\subsubsection{Participants and Organizer Tasks}

Participants are free to propose classifiers coupled to feature extraction of their choice. Their objective should be to optimize their approaches on both sets of development data.

Participants are required to send an executable code following precise guidelines that will be provided by the organizer. The organizer will provide comparative global 
results (on all 382 persons) as well as on categories of writers as defined in Section 3.3. The organizer will also test the executables proposed by the participants in conditions of time variability, thanks to the protocol given in Section 3.4.2.

Up to now, 11 academic participants are registered to BSEC'2009.

\section{Conclusion}

We have presented in this paper two competitions that are on-going, on fingerprints and on-line signatures. Both present original protocols which are representative of the actual state of the art in their domain and which will provide interesting comparative results. FVC on-going will be officially launched at ICB 2009 in June while the results of BSEC 2009 will be presented during the conference.

\section{References}

1. Maio, D., Maltoni, D., Cappelli, R., Wayman, J.L., Jain, A.K.: FVC 2000: Fingerprint Verification Competition. IEEE Transactions on Pattern Analysis Machine Intelligence 24(3), 402-412 (2002)

2. Maio, D., Maltoni, D., Cappelli, R., Wayman, J.L., Jain, A.K.: FVC 2002: Second Fingerprint Verification Competition. In: Proceedings 16th International Conference on Pattern Recognition (ICPR 2002), Québec City, August 2002, vol. 3, pp. 811-814 (2002)

3. Cappelli, R., Maio, D., Maltoni, D., Wayman, J.L., Jain, A.K.: Performance Evaluation of Fingerprint Verification Systems. IEEE Transactions on Pattern Analysis Machine Intelligence 28(1), 3-18 (2006)

4. Cappelli, R., Ferrara, M., Franco, A., Maltoni, D.: Fingerprint verification competition 2006. Biometric Technology Today 15(7-8), 7-9 (2007)

5. FVC-onGoing web site, http://bias.csr.unibo.it/fvcongoing/ (accessed, November 2008)

6. Yeung, D.Y., Chang, H., Xiong, Y., George, S., Kashi, R., Matsumoto, T., Rigoll, G.: SVC 2004: First international signature verification competition. In: Zhang, D., Jain, A.K. (eds.) ICBA 2004. LNCS, vol. 3072, pp. 16-22. Springer, Heidelberg (2004)

7. http://www.biosecure. info

8. http://www. int-evry.fr/biometrics/BMEC2007

9. http://biometrics.it-sudparis.eu/BSEC2009/

10. Garcia-Salicetti, S., Houmani, N., Dorizzi, B.: A Client-entropy Measure for On-line Signatures. In: Proceedings of IEEE Biometrics Symposium (BSYM), Tampa, USA, September 2008, pp. 83-88 (2008)

11. Houmani, N., Garcia-Salicetti, S., Dorizzi, B.: A Novel Personal Entropy Measure confronted with Online Signature Verification Systems Performance. In: Proceedings of IEEE Second International Conference on Biometrics: Theory, Applications and Systems (BTAS), Washington (September 2008)

12. Rabiner, L., Juang, B.H.: Fundamentals of Speech Recognition. Prentice Hall Signal Processing Series (1993) 